\title{
Cancer-associated Dermatomyositis: Does the PD-1 Checkpoint Pathway Play a Role?
}

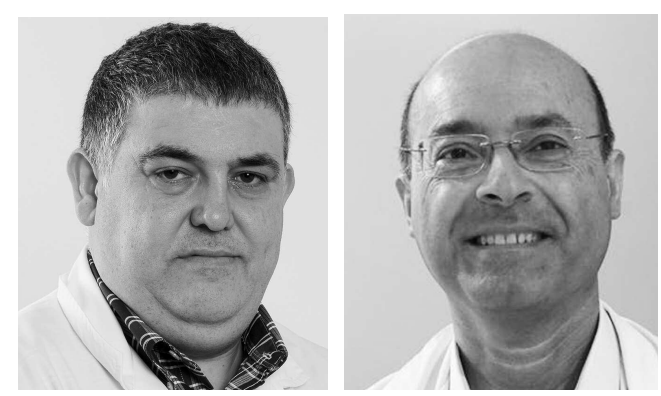

Cancer-associated myositis represents a unique opportunity to study the complex relationship between cancer and autoimmunity. It is well recognized that dermatomyositis (DM) is the inflammatory myopathy most often associated with cancer. In clinical practice, nearly one-third of patients with DM present with associated malignant disease, and the remainder may harbor an occult cancer that could develop in the near future, or never appear ${ }^{1,2}$. The immune system likely has a role in determining which of these outcomes will ensue.

Management of a patient with DM is difficult, because an occult malignancy may not always be detected by the available screening tools. One explanation for the uncertainty regarding the development of malignant disease in these patients is the concept of 3 sequential phases in the relationship between cancer and the immune system: elimination, equilibrium, and escape ${ }^{3}$. In this framework, patients with cancer-associated DM can be viewed in different ways. First, they can be seen as DM patients with concurrent malignant disease, which would indicate that elimination of the malignancy by the immune system has failed. Second, the patients could have an occult malignancy, but the immune system maintains a tight balance to prevent it from full development to cancer. This is the equilibrium phase, which can last for decades and helps explain the higher incidence of cancer in patients with DM even years after the diagnosis, and the difficulty of detecting the cancer, because it is controlled at the molecular level by the immune system and no morphologic or functional changes are evident. Finally, the malignant disease can manifest as an unexpected full-blown cancer with metastasis and a poor prognosis, because of a progressive loss of immune control that allows tumor cells to escape immune surveillance. This immune control failure results from several mechanisms. There is less intense expression of tumor-specific antigens and a decreasing affinity for the HLA class I-related peptides that elicit the autoimmune response against these antigens. In addition, the inhibitory checkpoint pathway, represented by programmed death 1 /programmed death ligand 1 (PD-1/PD-L1), may be activated and lead to suppression of the antitumor response. Taken together, these are some of the mechanisms implicated in the escape of tumor cells from the immune system.

Two particular articles have focused on this topic. A group from Barcelona recently published a report investigating genetic changes in the tumors of patients with cancer-associated $\mathrm{DM}^{4}$. A significant number of alterations were described, including mutations and loss of heterozygosity in patients with cancer-associated DM testing positive for antibodies against antitranscriptional intermediary factor $1-\gamma($ TIF1- $\gamma)$, an acknowledged biomarker of malignancy in these patients ${ }^{5,6}$, compared to DM controls negative for the antibody and cancer. The proposed hypothesis was that mutations in TIF1 proteins in the tumor, a well-described phenomenon, induce a strong immune response against the mutated antigen, and this can lead to 2 different situations. The first would be elimination of all neoantigens and tumor cells, and in this case, from the clinical viewpoint, the patient with DM is free from malignancy. In the second scenario, tumor cells try to escape from the immune attack by a loss of heterozygosity; that is, by losing the mutated gene. If they succeed, undifferentiated malignant tumor cells survive, and this leads to the development of an aggressive cancer that is recognized in clinical practice as unexpected full-blown malignant disease, with an unfavorable outcome. A similar mechanism has been suggested in patients with systemic sclerosis and cancer ${ }^{7}$.

The second article, by Chen, $e t a l^{8}$ in this issue of The Journal, reports an interesting study. The authors investigated serum concentrations of soluble PD-L1 (sPD-L1) in several groups of patients with DM: those without cancer, and patients with associated cancer divided into 2 groups, 1 with recent-onset therapy-naive cancer and the other with stable cancer in remission or cured by surgery or standard chemotherapy. The control groups included healthy controls, patients without DM diagnosed with solid tumors, and

See sPD-L1 and malignancy in DM, page 835

Personal non-commercial use only. The Journal of Rheumatology Copyright @ (2018. All rights reserved. 
patients with other autoimmune diseases such as systemic lupus erythematosus or rheumatoid arthritis. What they found was interesting: non-DM patients with solid tumors had the highest SPD-L1 values and healthy individuals the lowest. These findings are logical and reasonable. But what was seen in the groups of patients with DM? Those with DM without cancer had mildly elevated values, more or less similar to the values found in patients with other autoimmune disorders, whereas those with treatment-naive cancer had significantly higher values than patients with DM without cancer or those with stable cancer.

For the diagnosis of cancer-associated DM, the authors suggested an SPD-L1 cutoff that had an area under the curve of 0.72 , which is considered only fair for diagnostic purposes. However, when this value was combined with positive testing for anti-TIF1- $\gamma$ antibody (which has a very high negative predictive value $^{6}$ ), the specificity and positive predictive value of the combined variables increased to $95 \%$ and $70 \%$, respectively, and yielded a diagnostic capability greater than that of anti-TIF1- $\gamma$ alone for cancer screening in patients with DM. Therefore, the combination of TIF $1-\gamma$ antibody and sPD-L1 testing may be the best option for cancer detection in this population.

The data reported by Chen, et $a l^{8}$ are congruent. PD-1 inhibitors have been used in cancer therapy with good outcomes, the rationale being to enhance the immune response against tumor cells ${ }^{9}$. The PD- 1 checkpoint pathway is considered a physiologic immune regulatory pathway that allows self-tolerance to our own antigens by downregulation of the immune response. Blockade of this pathway can produce paradoxical adverse autoimmune phenomena, such as immune-mediated myopathies ${ }^{10,11}$. Serum sPD-L1, which is released from PD-L1-positive cells and binds to the PD-1 receptor, contributes to immunoregulation and can be considered a good surrogate of activation of this pathway. Moreover, sPD-L1 seems to play a role in promoting cancer cells, as was recently reported in a metaanalysis on this topic, in which it emerged as a biomarker of an unfavorable prognosis in patients with cancer ${ }^{12}$. Therefore, in what is likely a multistep process or a concurrent action, these mechanisms - cancer cell mutations and loss of heterozygosity on the one hand and PD-1 pathway activation on the other - participate in the complex relationship between cancer and the immune system, here represented by DM, an autoimmune disease.

Although the results obtained by Chen, et $a l^{8}$ must be reproduced and confirmed in other DM cohorts and in patients of other ethnicities, the study should be considered a step forward that empowers the clinician with a means for more accurate cancer screening and enhances our knowledge of the cancer-DM relationship. Most importantly, improving our understanding of the mechanisms that underlie these entities will enable us to provide better care for our patients.
MOISES LABRADOR-HORRILLO, $\mathrm{MD}, \mathrm{PhD}$ Associate Professor; ALBERT SELVA-O'CALLAGHAN, MD, $\mathrm{PhD}$, Professor,

Systemic Autoimmune Diseases Unit, Vall D'Hebron General Hospital, Internal Medicine Department, Universitat Autonoma de Barcelona, Barcelona, Spain.

Address correspondence to Dr. M. Labrador-Horrillo, Vall d'Hebron General Hospital, Internal Medicine, Passeig Vall d'Hebron, 119-129 Barcelona, 08035 Spain.E-mail: mlabrador@vhebron.net. Work funded by the Instituto de Salud Carlos III, grant PI15-02100, co-financed by the European Regional Development Fund.

\section{REFERENCES}

1. Selva-O'Callaghan A, Trallero-Araguás E, Grau-Junyent JM, Labrador-Horrillo M. Malignancy and myositis: novel autoantibodies and new insights. Curr Opin Rheumatol 2010;22:627-32.

2. Aussy A, Boyer O, Cordel N. Dermatomyositis and immune-mediated necrotizing myopathies: a window on autoimmunity and cancer. Front Immunol 2017;8:992.

3. Schreiber RD, Old LJ, Smyth MJ. Cancer immunoediting: integrating immunity's roles in cancer suppression and promotion. Science 2011;331:1565-70.

4. Pinal-Fernandez I, Ferrer-Fabregas B, Trallero-Araguas E, Balada E, Martínez MA, Milisenda JC, et al. Tumour TIF1 mutations and loss of heterozygosity related to cancer-associated myositis. Rheumatology 2017 Nov 14 (E-pub ahead of print)

5. Trallero-Araguás E, Rodrigo-Pendás JÁ, Selva-O'Callaghan A, Martínez-Gómez X, Bosch X, Labrador-Horrillo M, et al. Usefulness of anti-p155 autoantibody for diagnosing cancer-associated dermatomyositis: a systematic review and meta-analysis. Arthritis Rheum 2012;64:523-32.

6. Labrador-Horrillo M, Martínez MA, Selva-O'Callaghan A, Trallero-Araguás E, Balada E, Vilardell-Tarrés M, et al. Anti-TIF1 $\gamma$ antibodies (anti-p155) in adult patients with dermatomyositis: comparison of different diagnostic assays. Ann Rheum Dis 2012;71:993-6

7. Joseph CG, Darrah E, Shah AA, Skora AD, Casciola-Rosen LA, Wigley FM, et al. Association of the autoimmune disease scleroderma with an immunologic response to cancer. Science 2014;343:152-7.

8. Chen H, Peng Q, Yang H, Yin L, Shi J, Zhang Y, et al. Increased levels of soluble programmed death ligand 1 associate with malignancy in patients with dermatomyositis. J Rheumatol 2018;45:835-40.

9. Boussiotis VA. Molecular and biochemical aspects of the PD-1 checkpoint pathway. N Engl J Med 2016;375:1767-78.

10. Abdel-Wahab N, Shah M, Suarez-Almazor ME. Adverse events associated with immune checkpoint blockade in patients with cancer: a systematic review of case reports. PLoS One 2016;11:e0160221

11. Liewluck T, Kao JC, Mauermann ML. PD-1 inhibitor-associated myopathies: emerging immune-mediated myopathies. J Immunother 2017 Dec 1 (E-pub ahead of print).

12. Ding Y, Sun C, Li J, Hu L, Li M, Liu J, et al. The prognostic significance of soluble programmed death ligand 1 expression in cancers: a systematic review and meta-analysis. Scand J Immunol 2017;86:361-7.

J Rheumatol 2018;45:731-2; doi:10.3899/jrheum.180007

Personal non-commercial use only. The Journal of Rheumatology Copyright $\subset$ $\subset$ 2018. All rights reserved 